\title{
Variacion estacional del abastecimiento de frutales nativos de colecta en dos épocas climáticas en el mercado de Pucallpa
}

\section{Seasonal variation in the supply of native collected fruits in two weather seasons in the Pucallpa market}

\author{
(iD) 'Ena Vilma Velazco Castro (iD '2Julio Alegre Orihuela (iD ' Ronald Marlon Lozano Reátegui \\ (iD) 'José Antinori Samán (iD) 'Jessy Isabel Vargas Flores (iD 'Cindy Paola Castro Muñoz \\ (iD) 'Mariano Magdaleno Mendoza Carlos
}

Universidad Nacional Intercultural de la Amazonia. Pucallpa - Perú. ${ }^{1}$ Universidad Agraria La Molina. Lima - Perú

\section{Resumen}

El movimiento de comercialización de frutales nativos en la región Ucayali es muy intenso y no existe un inventario de flujos de comercialización de productores e intermediarios, quienes regulan precios y distribución a diferentes niveles. Este estudio tuvo como objetivo generar información sobre la variación estacional del abastecimiento de frutales nativos de colecta en dos épocas climáticas en el mercado de Pucallpa entre abril a diciembre 2017. Se aplicó encuesta semiestructurada a colectores de frutales nativos, asimismo, se levantó información diaria sobre la cantidad de colectores que acudían al mercado. Las variables medidas fueron: estacionalidad, volumen, lugares de colecta y relación de las condiciones climáticas frente a la estacionalidad de los frutales nativos. Los resultados indican que en la época climática seca existe mayor abundancia de Mauritia flexuosa (30,8 \%), mayor volumen de Solanum sessiliflorum (271,1 toneladas). Los lugares de colecta con mayor frecuencia correspondieron a Región Ucayali, Provincia Coronel Portillo, Distritos Calleria, Campo Verde y Yarinacocha. Hubo correlación alta entre precipitación y Solanum sessiliflorum ( $r$ :0.956). La tendencia sobre la presencia de frutales nativos en el mercado fue variable, con mayor presencia de Mauritia flexuosa (30,8 \%) e Inga edulis (22,5\%) en época climática seca y lluviosa respectivamente.

Palabras clave: Frutales nativos, época climática, variación, estacionalidad.

Recibido: Junio 2020

Aceptado: Octubre 2020

\section{Abstract}

The commercialization movement of native fruit trees in the Ucayali region is very intense and there is no inventory of commercialization flows from producers and intermediaries, who regulate prices and distribution at different levels. The objective of this study was to generate information on the seasonal variation of 
the supply of native fruit trees for collection in two climatic seasons in the Pucallpa market between April and December 2017. A semi-structured survey was applied to collectors of native fruit trees, as well as daily information on the number of collectors who came to the market. The variables measured were: seasonality, volume, places of collection and relation of the climatic conditions against the seasonality of the native fruit trees. The results indicate that in the dry climatic season there is a greater abundance of Mauritia flexuosa (30.8\%), a greater volume of Solanum sessiliflorum (271.1 tons). The most frequent collection sites corresponded to the Ucayali Region, Coronel Portillo Province, Districts Calleria, Campo Verde and Yarinacocha. There was a high correlation between precipitation and Solanum sessiliflorum ( $r$ : 0.956). The trend regarding the presence of native fruit trees in the market was variable, with a greater presence of Mauritia flexuosa (30.8\%) and Inga edulis (22.5\%) in dry and rainy weather respectively.

Keywords: Native fruit trees, climatic season, variation, seasonality.

\section{INTRODUCCIÓN}

La Amazonía es una de las regiones mega diversas del planeta, debido a los diversos procesos naturales que han originado la cuenca Amazónica, las mismas que determinaron la variabilidad ecológica y por ende la riqueza florística y es así que, en la flora fanerógama de la Amazonia peruana se registraron 6237 especies, el cual representa el 36.3\% del total de especies en el Perú, distribuidos en 1406 géneros, 182 familias y 650 especies endémicas (Clavo., 2010).

En referencia a los frutales nativos, Brack (2003), menciona que en el Perú existen 553 especies de frutales nativos. Mientras que Gonzales (2007), reportó que en la región Loreto se consumen por lo menos 193 especies de frutos, 139 cosechados de las poblaciones naturales de los cuales en los mercados de la ciudad de lquitos se comercializan frutos de 60 especies y 21 familias; de estos, los frutos de 41 especies provienen de cosecha de las poblaciones silvestres. Asimismo, para Ucayali, Guillén (2010), realizó una colección local de germoplasma de frutales tropicales encontrando 28 especies con 64 accesiones. En ese mismo sentido, Baltazar (2011) registra 24 especies de frutales nativos como resultado de su estudio realizado el año 2009 y 2010.

Por su lado, Huamantupa (2008), aproximó a 320 especies de frutales nativos que se con- sumen entre los departamentos de Cuzco, Loreto y Madre de Dios, comercializándose ampliamente en el mercado local las especies: Mauritia flexuosa L. f, Solanum sessiliflorum Dunal y Psidium guajava L. Asimismo, la riqueza específica está distribuida en 46 familias y 131 géneros, las familias con más abundancia en especies son Arecaceae (28 especies), Fabaceae (25), Moraceae y Malvaceae (16) y Myrtaceae (15). El mismo autor reporta una variación estacional de los frutales nativos en el periodo comprendido entre marzo y junio con mayor abundancia (85 y 125 especies de frutales nativos por día), mientras que en las temporadas secas o de verano disminuye, llegando a solo 30 especies por día.

Por su parte, Mostacero et al., (2017), sobre frutales nativos del norte del Perú, reportaron 45 especies, distribuidas en 18 familias, por su número destacan: Ericaceae (7), Myrtaceae (6), Cactaceae (4), Solanaceae (4), Caricaceae (3), Passifloraceae (3) y Rosaceae (3).

Asimismo, Mitjans et al., 2019, identificaron 17 especies de frutales nativos, distribuido en 14 familias y 17 géneros, la familia con más especies representativas fue Malvaceae.

Por consiguiente, existe alta diversidad de frutales nativos que necesitan ser estudiados en el aspecto agronómico, comercialización, 
producción, estacionalidad, volúmenes de extracción y valor agregado de las mismas.

Los frutos nativos amazónicos se caracterizan por ser numerosos, con composición nutricional variada, con estacionalidad de producción variada, que comprende los doce meses del año y presenta alternativas de consumo humano y/o animal. Sin embargo, aún no existen datos sistematizados como producción, estacionalidad, volumen, lugares de colecta, usos y comercialización en los mercados, de los frutales nativos para la ciudad de Pucallpa en todo el año, por lo tanto, existe un vacío y poca información estadística confiable de los extensionistas, que permita tener datos exactos sobre el comportamiento y presencia de los frutales nativos a través del año en el mercado de Pucallpa.

Por ello se ha planteado el siguiente objetivo generar información sobre la variación estacional del abastecimiento de frutales nativos de colecta en dos épocas climáticas en el mercado de Pucallpa.

\section{MATERIALES Y MÉTODOS}

Diseño de la investigación: El estudio se ajustó a un diseño no experimental de tipo longitudinal: porque fue una investigación sistemática y empírica en la que la variable de interés solo fue observada tal y como ocurrió en su contexto natural. Y el tipo longitudinal porque se colectó datos durante nueve meses, remarcando las dos épocas climáticas.

Población y muestra: La población fueron todos los colectores de frutales nativos que llegaron al Mercado de Productores 23 de noviembre. La muestra fueron 20 colectores de frutales nativos. El tipo de muestreo fue no probabilístico de tipo intencional.

Ubicación del lugar de estudio: El estudio se desarrolló en el Mercado de Productores 23 de noviembre, lugar donde concurren los colectores de frutales nativos a vender sus productos.
Geográficamente el mercado se ubica en la Región Ucayali, Provincia de Coronel Portillo, Distrito de Manantay, lugar específico entrando $2 \mathrm{~km}$ a la margen izquierda, a la altura del km 6 de la Carretera Federico Basadre - CFB.

Identificación al segmento a encuestar: Dentro del Mercado de Productores Regionales 23 de noviembre, existe un espacio de 840 $\mathrm{m}^{2}$, donde diariamente arriban los colectores de frutales nativos para expender sus productos de manera temporal, sólo hasta que terminen de vender (no superan las seis horas).

Descripción y técnicas e instrumentos de recolección de datos: La técnica de recolección de datos fue directa, a través de un instrumento (cuestionario).

Elaboración del cuestionario: De acuerdo a los objetivos de la investigación se elaboró un cuestionario con preguntas abiertas y cerradas, para obtener los datos de los colectores que traen los frutales nativos hacia el mercado de productores 23 de noviembre. Considerando preguntas como: tipo de frutal nativo, cantidad, lugar de procedencia, vía de acceso para transporte, percepción sobre el comportamiento del frutal nativo frente al cambio climático. Así como, la estacionalidad: riqueza de especies, abundancia de especies, índice de diversidad de especies (Shannon) e índice de diversidad de Simpson (1-D); volumen de frutales nativos que llegan al mercado (cálculo de la cantidad expresado en toneladas/ mes y época climática); lugares de colecta de los frutales nativos: frecuencia absoluta por región, provincia, distrito y caserío.

Prueba piloto: El cuestionario se sometió a una prueba piloto, lo que permitió realizar ajustes y modificaciones a las preguntas, esta actividad se realizó entre enero y febrero 2017.

Determinación de la época climática: El estudio se desarrolló, considerando las dos épocas climáticas, que presenta Ucayali, que según la DRAU (2012), son las siguientes: 
- Época seca: junio a noviembre

- Época lluviosa: diciembre, abril y mayo

Aplicación de la encuesta: Se encuestó a 20 colectores mensualmente, quienes Ilegaron al mercado trayendo los frutales nativos, sólo los días domingos a partir de las 6:00 am.

Registro de colectores diario: Se realizó un registro diario de ingreso de colectores al Mercado de Productores 23 de noviembre, debido a que no existe una data de la cantidad exacta que ingresan, ni los frutales nativos que vienen a vender. Esta actividad se realizó partir del 17.04.2017 y concluyó el 31.12.2017; los registros se realizaban a partir de las 5:00 am todos los días.
Tratamiento estadístico: Ios datos se analizaron mediante la hoja de cálculos EXCEL. En este estudio los resultados fueron analizados haciendo uso de estadística descriptiva y correlación de Pearson, para relacionar las condiciones climáticas frente a la estacionalidad de los frutales nativos. Se consideró un nivel de significancia estadístico p:<0.05.

\section{RESULTADOS Y DISCUSIÓN}

Estacionalidad de la presencia de frutales nativos en el mercado de Pucallpa: El Mercado de Productores 23 de noviembre, recibe mensualmente un promedio de 2714 colectores de frutales nativos, comercializando durante el año: 20 especies, distribuidos en 17 géneros, 12 familias y 11 órdenes (Tabla 1).

\section{Tabla 1} Total de especies frutales nativos presentes en el Mercado de Productores 23 de
noviembre según orden, familia, género y especie (abril-diciembre 2017)

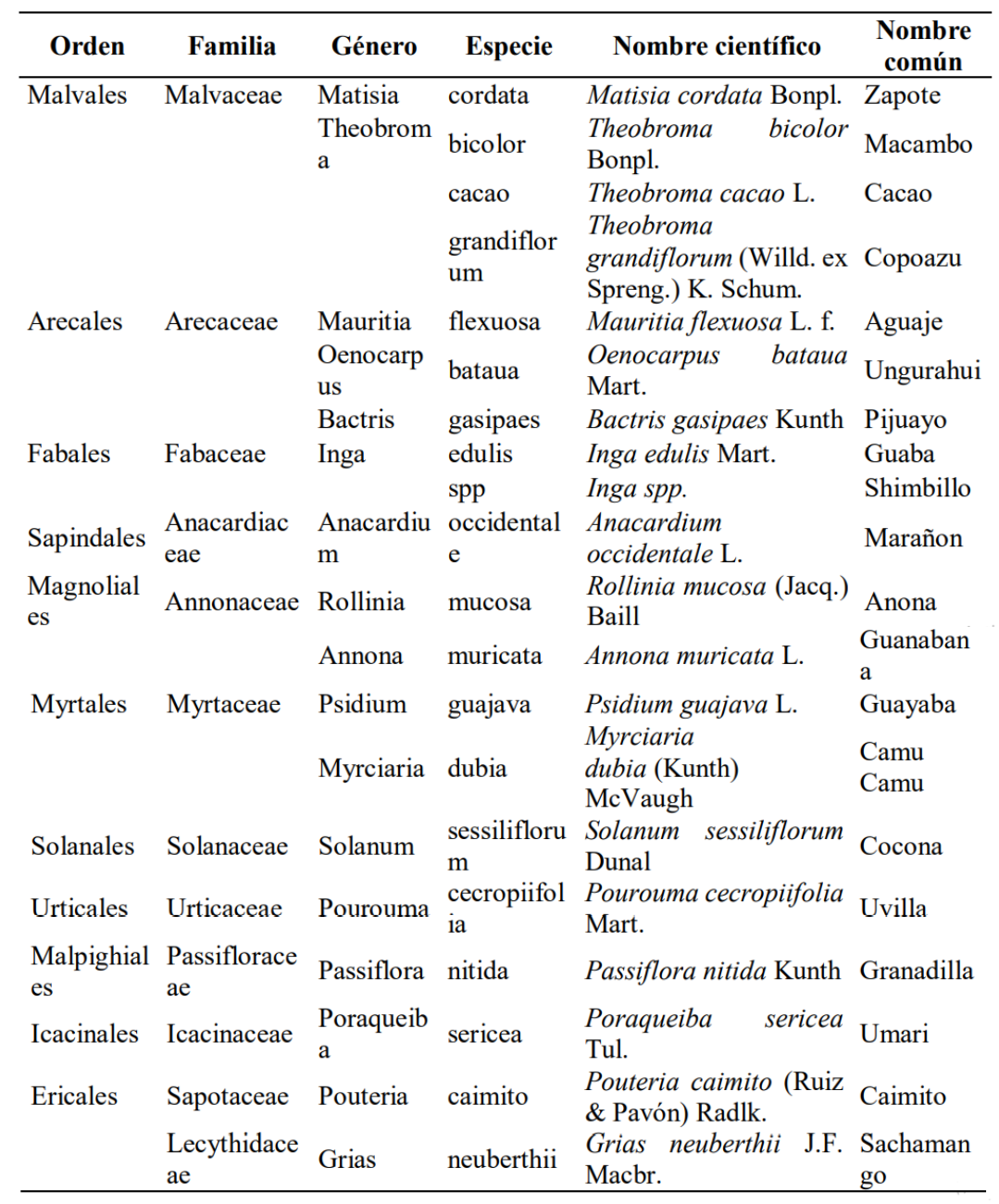


Al comparar los datos de este estudio con Baltazar (2011) y Guillén (2010) para Ucayali, se registra una tendencia negativa de la pérdida de la biodiversidad de frutales nativos, oscilando entre $20 \%$ y $40 \%$ respecto al presente estudio. Esta disminución, de la presencia de frutales nativos, estaría relacionada con los efectos del cambio climático, como lo menciona FAO (2008), cuando señala que las variaciones en las temperaturas y las precipitaciones; así como, la mayor frecuencia de condiciones meteorológicas extremas, determinará reducciones de la producción agropecuaria y pérdidas de otros bienes, que pondrán en peligro no sólo la producción de alimentos, sino también el acceso a los recursos alimentarios a la estabilidad y la utilización de los mismos.

Al describir la presencia de frutales nativos por época climática, en la Tabla 2 se muestra que en la época climática lluviosa (diciembre - mayo) están presentes cuatro especies: Inga edulis Mart., Mauritia flexuosa L. f., Myrciaria dubia (Kunth) McVaugh y Solanum sessiliflorum Dunal, quienes componen el 61,6\% del total de frutales nativos que ingresan al mercado. Asimismo, en la época climática seca (junio-noviembre), están presentes tres especies Myrciaria dubia (Kunth) McVaugh, Mauritia flexuosa L. f. y Solanum sessiliflorum Dunal, que en conjunto componen el $62,4 \%$, de frutales nativos que los colectores llevan al mercado.

\section{Tabla 2}

Total, y porcentaje de especies de frutales nativos presentes en el Mercado de Productores 23 de noviembre por época climática (abril-diciembre 2017)

\begin{tabular}{|c|c|c|c|c|c|}
\hline \multirow{3}{*}{ Nombre científico } & \multirow{3}{*}{$\begin{array}{l}\text { Nombre } \\
\text { común }\end{array}$} & \multicolumn{4}{|c|}{ Época climática } \\
\hline & & \multicolumn{2}{|c|}{ Lluviosa } & \multicolumn{2}{|c|}{ Seca } \\
\hline & & $\mathrm{n}$ & $\%$ & $\mathrm{n}$ & $\%$ \\
\hline Inga edulis Mart. & Guaba & 309 & 22,5 & 130 & 3,2 \\
\hline Mauritia flexuosa $\mathrm{L}$. f. & Aguaje & 199 & 14,5 & 1234 & 30,8 \\
\hline $\begin{array}{l}\text { Myrciaria } \quad \text { dubia (Kunth) } \\
\text { McVaugh }\end{array}$ & Camu Camu & 185 & 13,5 & 778 & 19,4 \\
\hline Solanum sessiliflorum Dunal & Cocona & 153 & 11,1 & 488 & 12,2 \\
\hline Passiflora nitida Kunth & Granadilla & 118 & 8,6 & 21 & 0,5 \\
\hline Oenocarpus bataua Mart. & Ungurahui & 102 & 7,4 & 406 & 10,1 \\
\hline Pourouma cecropiifolia Mart. & Uvilla & 53 & 3,9 & 168 & 4,2 \\
\hline Matisia cordata Bonpl. & Zapote & 53 & 3,9 & 4 & 0,1 \\
\hline $\begin{array}{l}\text { Pouteria caimito (Ruiz \& } \\
\text { Pavón) Radlk. }\end{array}$ & Caimito & 46 & 3,4 & 369 & 9,2 \\
\hline Theobroma bicolor Bonpl. & Macambo & 42 & 3,1 & 72 & 1,8 \\
\hline Theobroma cacao L. & Cacao & 39 & 2,8 & 64 & 1,6 \\
\hline Bactris gasipaes Kunth & Pijuayo & 22 & 1,6 & 128 & 3,2 \\
\hline $\begin{array}{l}\text { Theobroma grandiflorum } \\
\text { (Willd. ex Spreng.) K. Schum. }\end{array}$ & Copoazu & 19 & 1,4 & 23 & 0,6 \\
\hline Rollinia mucosa (Jacq.) Baill & Anona & 10 & 0,7 & 9 & 0,2 \\
\hline Inga spp. & Shimbillo & 9 & 0,7 & 2 & 0,0 \\
\hline Poraqueiba sericea Tul. & Umari & 7 & 0,5 & 2 & 0,0 \\
\hline Psidium guajava $\mathrm{L}$. & Guayaba & 3 & 0,2 & 3 & 0,1 \\
\hline Anacardium occidentale L. & Marañon & 3 & 0,2 & 91 & 2,3 \\
\hline Grias neuberthii J.F. Macbr. & Sachamango & 1 & 0,1 & 0 & 0,0 \\
\hline Annona muricata L. & Guanabana & 0 & 0,0 & 13 & 0,3 \\
\hline Número total de frutales (n) & & 1373 & 100,0 & 4005 & 100,0 \\
\hline Número total de especies (S) & & 19 & & 19 & \\
\hline
\end{tabular}


Respecto al mayor porcentaje de Inga edulis Mart, en la época climática lluviosa, coincide con Villachica (1996), quién menciona que la mayor producción de esta especie ocurre entre abril y mayo. Sobre Mauritia flexuosa L. f. Gonzales (2007), señala que esta especie se encuentra presente durante todo el año con mayor abundancia entre mayo a setiembre, mientras que Flores (1996), señala que hay mayor producción de Myrciaria dubia (Kunth) McVaugh y Solanum sessiliflorum Dunal, entre los meses de noviembre a mayo y abril a octubre, respectivamente, concordando con los reportes del presente estudio, el cual corresponde a la época climática seca. Sin embargo, se difiere con Huamantupa (2008), quién refiere que la mayor abundancia de frutales nativos se presenta entre marzo a junio (época lluviosa), y que en la temporada seca disminuyen estas frutas, puede deberse según el autor, el cual trabajó con tres departamentos Loreto, Cuzco y Madre de Dios, que fisiográficamente difiere con las condiciones climáticas de Ucayali.

De igual manera, los datos obtenidos a través del tiempo que duró el estudio, fueron analizados por los índices de diversidad de Shannon $\left(H^{\prime}\right)$ y Simpson (1-D), como se puede evidenciar en la Tabla 3, que durante la época climática lluviosa (diciembre a mayo), se presenta mayores valores en los índices respectivos H': 3,405 y 1-D: 0,878, ambos índices indican que existe una diversidad alta en la época climática lluvioso (Villachica 2006; Flores 1996; Gonzales 2007). La abundancia de especies osciló desde 1373 hasta 4005, mientras que la riqueza de frutales nativos fue de 19 especies para las dos épocas climáticas en estudio (Tabla 3).

\section{Tabla 3}

\section{Índice de diversidad de Shannon ( $\left.\mathrm{H}^{\prime}\right)$, índice de diversidad de Simpson (1-D), abundancia y riqueza de especies de frutales nativos en el Mercado de Producto- res 23 de noviembre por época climática (abril-diciembre 2017)}

\begin{tabular}{cccccc}
\hline $\begin{array}{c}\text { Época } \\
\text { climática }\end{array}$ & $\begin{array}{c}\text { Abundancia } \\
\text { (N) }\end{array}$ & $\begin{array}{c}\text { Riqueza } \\
\text { (S) }\end{array}$ & $\begin{array}{c}\text { Índice de } \\
\text { diversidad } \\
\text { de } \\
\text { Shannon } \\
\left(\mathbf{H}^{\prime}\right)\end{array}$ & $\begin{array}{c}\text { Índice de } \\
\text { dominancia } \\
\text { de Simpson } \\
\text { (D) }\end{array}$ & $\begin{array}{c}\text { Índice de } \\
\text { diversidad } \\
\text { de } \\
\text { Simpson } \\
(\mathbf{1 - D})\end{array}$ \\
\hline $\begin{array}{c}\text { Lluviosa } \\
\text { Seca }\end{array}$ & 1373 & 19 & 3,405 & 0,122 & 0,878 \\
\hline
\end{tabular}

Volumen de los frutales nativos que llegan al mercado de Pucallpa:

En la Tabla 4, se muestra los mayores volúmenes (toneladas) de frutales nativos que ingresaron al Mercado de Productores 23 de noviembre, evidenciando que, en la época lluviosa, está presente Inga edulis Mart (16,17 t), Oenocarpus bataua Mart. (29,70 t), Matisia cordata Bonpl (2,98 t), Rollinia mucosa (Jacq.) Baill (1,12 t), Passiflora nitida Kunth (2,19 t), Theobroma bicolor Bonpl. (1,64 t) y Theobroma cacao L. (0,75 t), mientras que para la época seca, se registraron Solanum sessiliflorum Dunal (271 t), Myrciaria dubia (Kun- th) McVaugh (50,67 t), Pourouma cecropiifolia Mart. (3,41 t), Mauritia flexuosa L. f. (211,60 t) y Bactris gasipaes Kunth (2,56 t).

En orden descendente, los mayores volúmenes de frutales nativos fueron: Solanum sessiliflorum Dunal (271 t), Mauritia flexuosa L. f. (211,60 t), Myrciaria dubia (Kunth) McVaugh (50,67 t), Oenocarpus bataua Mart. (29,70 t) e Inga edulis Mart (16,17 t).

Tanto Gonzales (2007), Huamantupa (2008), Villachica (1996), Baltazar (2011), coinciden en 
señalar que Mauritia flexuosa L. f., es el frutal nativo que más se consume en la Amazonía Peruana.

Lugares de colecta de los frutales nativos que llegan al mercado de Pucallpa: La Región Ucayali (96,8\%), Provincia de Coronel Portillo (86,9\%), Distrito de Callería, (24,5\%), Yarinacocha $(25,5 \%)$ y Campo Verde $(25,0 \%)$, aportan con frutales nativos al Mercado de Productores 23 de noviembre (ver Figuras 1, 2 y 3). En total, fueron 64 caseríos de los cuales proceden los frutales nativos, en la Figura 4, se observa que los caseríos Porvenir (Callería), Yerbas buenas (Campo Verde), San Juan de Yarinacocha, San José de Yarinacocha y Padre Bernardo (Yarinacocha) son los que aportan con mayor presencia de frutales nativos.

\section{Tabla 4}

\section{Volumen de ingreso al Mercado de Productores 23 de noviembre de frutales} nativos por época climática (abril-diciembre 2017)

\begin{tabular}{|c|c|c|c|c|c|c|c|c|c|c|}
\hline \multirow{2}{*}{ Nombre científico } & \multirow{2}{*}{$\begin{array}{l}\text { Nombre } \\
\text { común }\end{array}$} & \multirow{2}{*}{$\begin{array}{c}\text { Unidad de } \\
\text { medida } \\
(\mathrm{UM})\end{array}$} & \multirow{2}{*}{$\begin{array}{c}\text { Equivalencia } \\
\text { en kg }\end{array}$} & \multirow[b]{2}{*}{ Abril $^{1}$} & \multirow[b]{2}{*}{ Mayo $^{1}$} & \multirow[b]{2}{*}{ Junio $^{2}$} & \multicolumn{4}{|c|}{ Volumen de ingreso (tonelada) } \\
\hline & & & & & & & Julio $^{2}$ & Agosto $^{2}$ & Septiembre $^{2}$ & Octubre $^{2}$ \\
\hline Inga edulis Mart. & Guaba & Ciento & 37,3 & 15,34 & 0,71 & 0,52 & 2,22 & 2,53 & - & - \\
\hline Matisia cordata Bonpl. & Zapote & Ciento & 37,3 & 2,91 & 1,96 & - & - & - & - & - \\
\hline $\begin{array}{l}\text { Solanum sessiliflorum } \\
\text { Dunal }\end{array}$ & Cocona & Saco & 65,0 & 1,24 & 2,99 & 271,15 & 152,65 & 259,84 & 126,75 & 21,45 \\
\hline $\begin{array}{l}\text { Rollinia mucosa (Jacq.) } \\
\text { Baill }\end{array}$ & Anona & Unidad & 1,2 & 1,12 & - & 0,16 & - & - & - & - \\
\hline Passiflora nitida Kunth & Granadilla & Ciento & 2,8 & 0,73 & 2,19 & 0,06 & 0,15 & - & - & - \\
\hline $\begin{array}{l}\text { Myrciaria dubia (Kunth) } \\
\text { McVaugh }\end{array}$ & Camu camu & Jaba & 25,0 & 0,70 & 23,63 & 50,67 & 8,50 & 9,10 & 10,10 & 7,92 \\
\hline $\begin{array}{l}\text { Pouteria caimito (Ruiz \& } \\
\text { Pavón) Radlk. }\end{array}$ & Caimito & Unidad & 0,2 & 0,07 & - & - & - & - & - & - \\
\hline Inga spp. & Shimbillo & Ciento & 4,3 & 0,02 & - & - & 0,04 & - & - & - \\
\hline Theobroma bicolor Bonpl. & Macambo & Unidad & 1,9 & 0,02 & 1,64 & 0,61 & 0,15 & - & - & - \\
\hline Poraqueiba sericea Tul. & Umari & Ciento & 0,1 & 0,004 & - & - & - & - & - & - \\
\hline Theobroma cacao L. & Cacao & Unidad & 0,6 & - & 0,75 & 0,13 & 0,03 & 0,01 & 0,14 & 0,35 \\
\hline Mauritia flexuosa L. f. & Aguaje & Saco & 60,0 & - & - & 7,65 & 76,15 & 170,40 & 29,88 & 211,60 \\
\hline Anacardium occidentale $\mathrm{L}$. & Marañon & Unidad & 0,1 & - & - & - & 0,03 & 0,04 & - & 0,23 \\
\hline Oenocarpus bataua Mart. & Ungurahui & Saco & 60,0 & - & - & - & - & 7,68 & 2,70 & 1,95 \\
\hline $\begin{array}{l}\text { Pourouma cecropiifolia } \\
\text { Mart. }\end{array}$ & Uvilla & Racimo & 0,5 & - & - & - & - & 0,29 & 3,41 & 0,14 \\
\hline Bactris gasipaes Kunth & Pijuayo & Racimo & 4,1 & - & - & - & - & - & 1,21 & 2,56 \\
\hline
\end{tabular}

${ }^{1}$ Época lluviosa ${ }^{2}$ Época seca

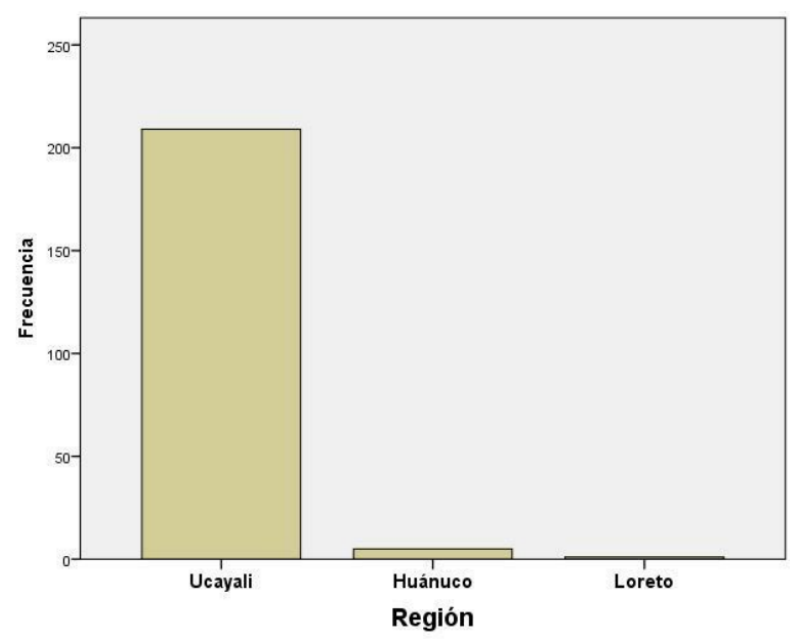

Figura 1. Región de procedencia de los frutales nativos 


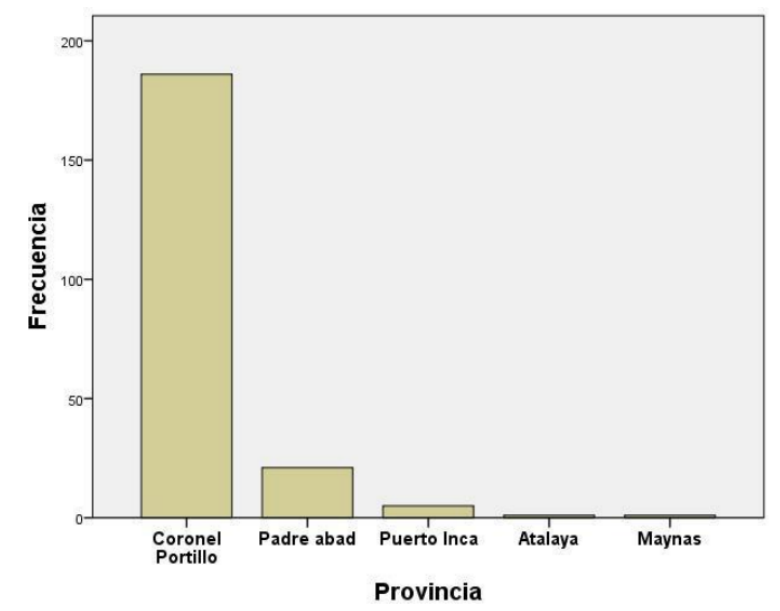

Figura 2. Provincia de procedencia de los frutales nativos.

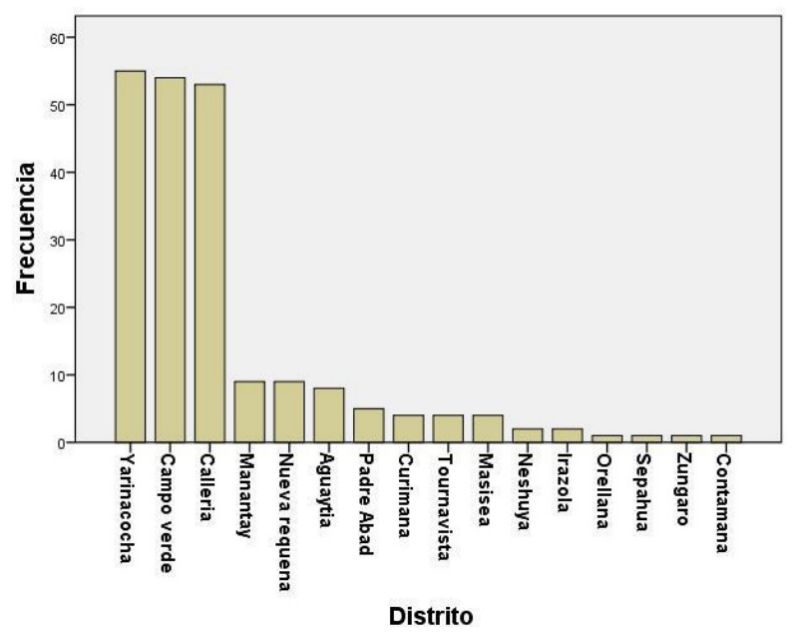

Figura 3. Distrito de procedencia de los frutales nativos.

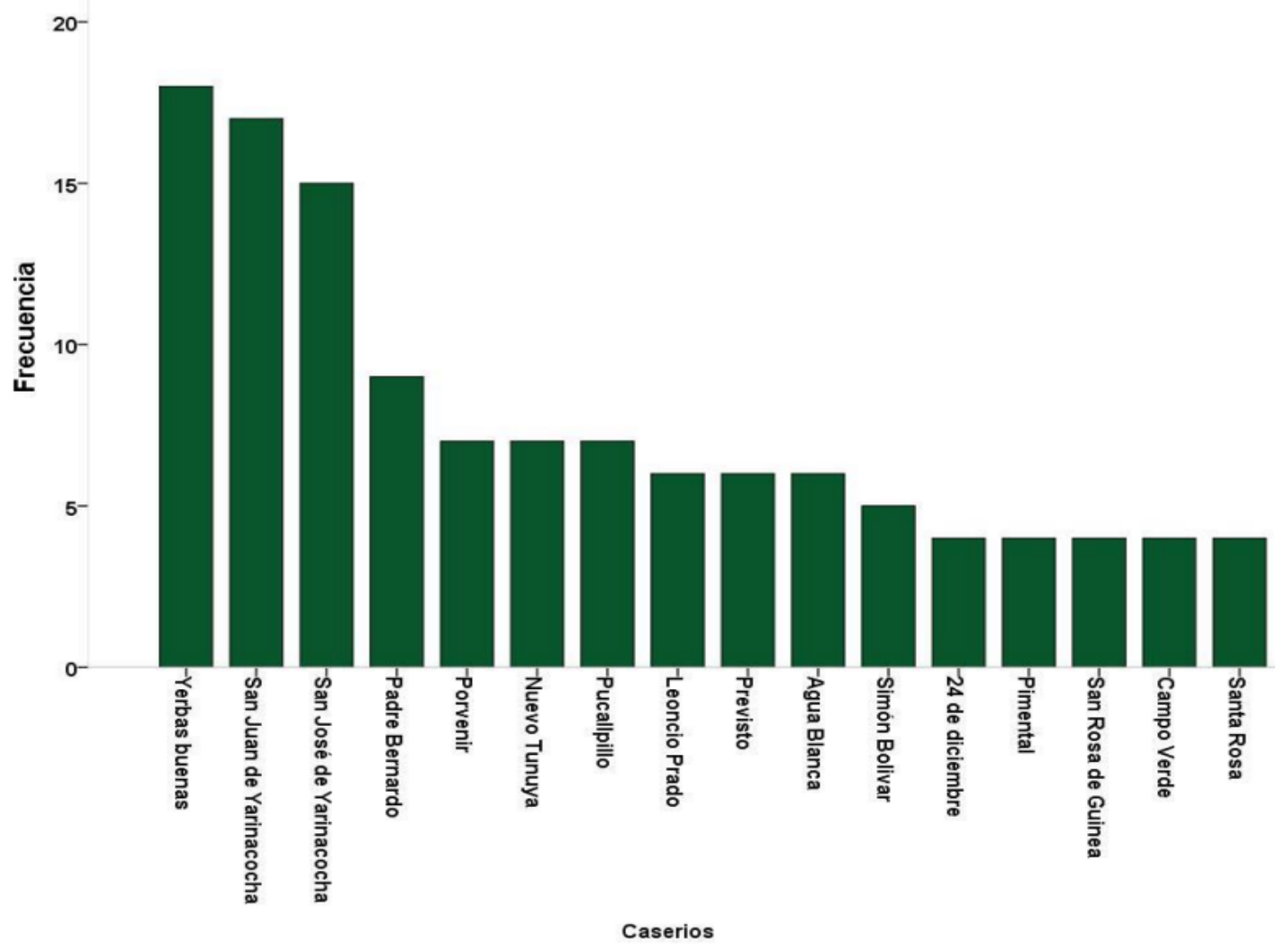

Figura 4. Caseríos de procedencia de los frutales nativos. 
Los cinco frutales nativos que llegan al Mercado de Productores 23 de noviembre en mayores volúmenes provienen de los caseríos
Yerbas buenas, San Juan de Yarinacocha, San José de Yarinacocha, Padre Bernardo y Porvenir (Figura 5).

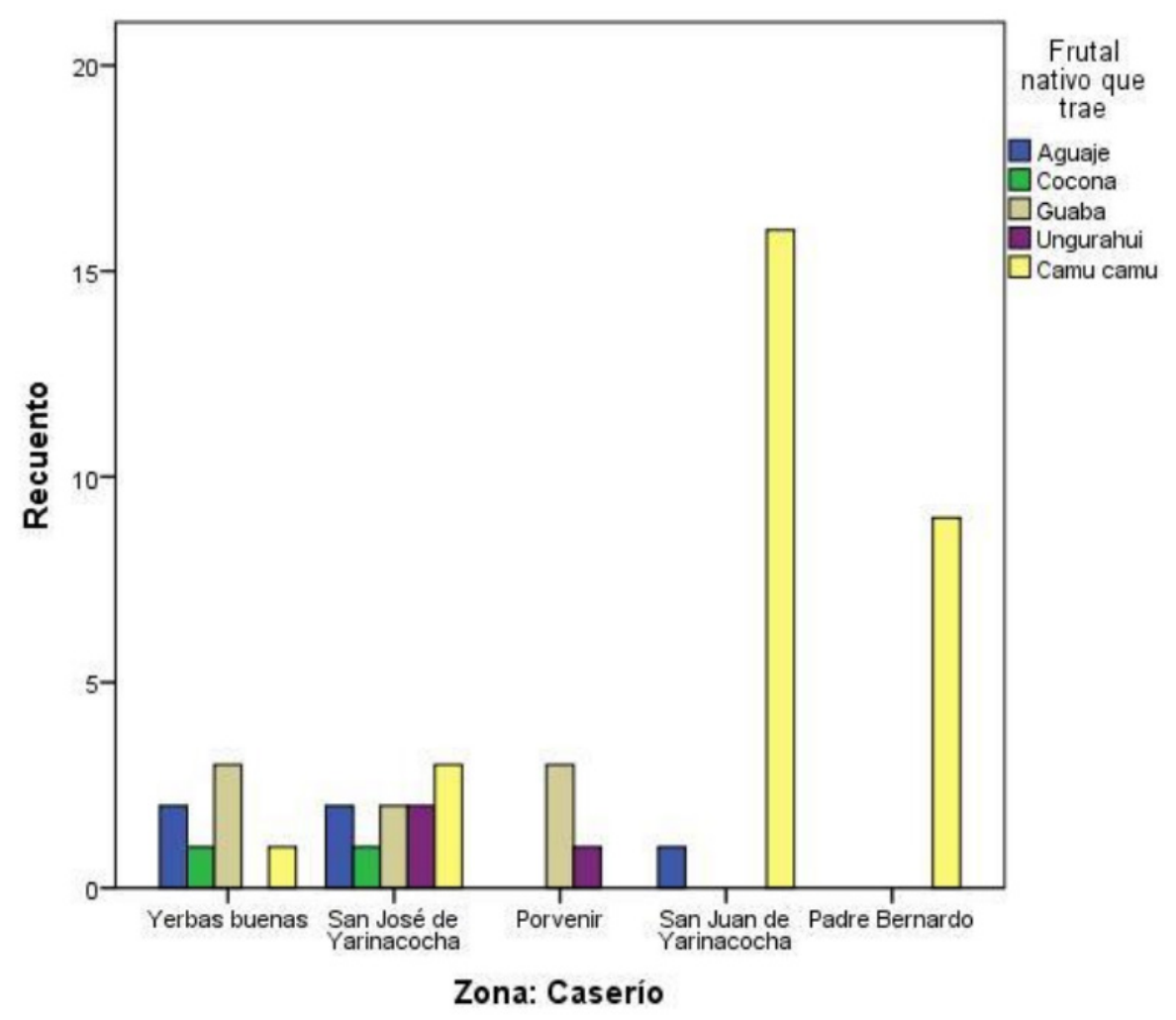

Figura 5. Caseríos de procedencia de los 5 frutales nativos con mayor volumen de ingreso al Mercado de productores 23 de noviembre.

Investigaciones realizadas por diversos autores con frutales nativos en la Amazonía, se puede resaltar que sobre Myrciaria dubia HBK Mc Vaugh"camu camu"Flores y Miranda (2017), reportaron que el 24\% de la producción procedente de los caseríos de Yarinacocha, son vendidos en los mercados locales, mientras que el $61 \%$ lo venden a intermediarios en la misma parcela y $15 \%$ lo entregan a empresas acopiadoras. Una experiencia sobre comercialización de Mauritia flexuosa "aguaje" señalado por Gamboa (2019) refiere que 67\% de los productores de aguaje realiza la venta en su propia chacra. Mientras que Arias et al., (2018), en su inves- tigación señalaron que el Oenocarpus bataua "ungurahui" son productos recolectados en la selva y que los excedentes pueden venderse a un creciente mercado de productos orgánicos y fair trade. En ese mismo sentido, para el poblador amazónico, Solanum sessiliflorum "cocona", tiene un valor económico, según lo reportado por Osores (2018). Los frutos de Inga edullis "guaba" es utilizado como alimento para las personas por el sabor dulce y carnoso del arilo (Collazos et al., 1995, citado por Castillo y Quija 2019). Relación de las condiciones climáticas frente a la estacionalidad de los frutales nativos en Pucallpa. 
Tabla 5

Correlación de Pearson de las condiciones climáticas con la presencia de frutales
nativos en el Mercado de Productores 23 de noviembre (abril-diciembre 2017)

\begin{tabular}{|c|c|c|c|c|c|c|}
\hline Frutales nativos & & $\begin{array}{l}\text { Temperatura } \\
\text { máxima } \\
\left({ }^{\circ} \mathrm{C}\right)\end{array}$ & $\begin{array}{l}\text { Temperatura } \\
\text { mínima }\left({ }^{\circ} \mathrm{C}\right)\end{array}$ & $\begin{array}{l}\text { Temperatura } \\
\text { media }\left({ }^{\circ} \mathrm{C}\right)\end{array}$ & $\begin{array}{l}\text { Precipitación } \\
(\mathrm{mm})\end{array}$ & $\begin{array}{c}\text { Humedad } \\
\text { relativa } \\
(\%)\end{array}$ \\
\hline \multirow{2}{*}{ Aguaje } & $\mathrm{r}$ &, $782^{*}$ &,- 130 & ,278 &,- 204 &,$- 704^{*}$ \\
\hline & $\mathrm{p}$ & ,013 & ,739 & ,469 & , 599 &, 034 \\
\hline \multirow{2}{*}{ Cacao } & $\mathrm{r}$ &,- 446 & ,084 &,- 168 & ,082 &, $677^{*}$ \\
\hline & $\mathrm{p}$ & ,229 &, 831 &, 666 & 833 &, 045 \\
\hline \multirow{2}{*}{ Cocona } & $\mathrm{r}$ &,- 304 &,$- 900^{* *}$ &,$- 800^{* *}$ &,$- 956^{* *}$ &,- 341 \\
\hline & $\mathrm{p}$ & ,427 &, 001 &, 010 &, 000 & 370 \\
\hline \multirow{2}{*}{ Copoazu } & $\mathrm{r}$ &,$- 736^{*}$ &,- 200 &,- 434 &,- 250 & ,471 \\
\hline & $\mathrm{p}$ &, 024 & ,606 & ,243 & ,516 & ,201 \\
\hline \multirow{2}{*}{ Guayaba } & $\mathrm{r}$ &,$- 680^{*}$ &,- 179 &,- 402 &,- 278 & ,434 \\
\hline & $\mathrm{p}$ &, 044 & ,644 & ,283 & ,470 & ,243 \\
\hline \multirow{2}{*}{ Marañon } & $r$ &, $698^{*}$ & ,092 & ,452 &, 026 &,- 294 \\
\hline & $\mathrm{p}$ &, 036 & ,813 & ,222 & 947 & ,443 \\
\hline \multirow{2}{*}{ Pijuayo } & $\mathrm{r}$ &, $786^{*}$ & ,305 & 620 & , 197 &,- 503 \\
\hline & $\mathrm{p}$ &, 012 & 425 & ,075 & 611 &, 167 \\
\hline \multirow{2}{*}{ Ungurahui } & $\mathrm{r}$ &, $819^{* *}$ & ,504 &, $765^{*}$ & ,397 &,- 398 \\
\hline & $\mathrm{p}$ &, 007 &, 167 &, 016 & 290, & 289 \\
\hline \multirow{2}{*}{ Uvilla } & $\mathrm{r}$ &, $671^{*}$ & 037 & ,364 &,- 061 &,$- 725^{*}$ \\
\hline & $\mathrm{p}$ &, 048 & ,926 & ,336 &, 876 &, 027 \\
\hline
\end{tabular}

*. p: $<0,05$ (2 colas). n: $9 ;$ r: correlación de

Pearson

**. p: $<0,01$ ( 2 colas).

En la Tabla 5, se muestra la correlación de los factores ambientales sobre la presencia de frutales nativos en el Mercado de Productores 23 de noviembre, evidenciando que la temperatura máxima tiene correlación significativa y negativa con copoazú y guayaba, correlación significativa y positiva con Marañon, Pijuayo, Ungurahui, y Uvilla, mientras que para la temperatura mínima, cocona presenta una correlación significativa y negativa; para la temperatura media, existe una correlación significativa alta y negativa con cocona y positiva con ungurahui, seguidamente la precipitación se correlaciona negativamente con cocona. Asimismo, aguaje y uvilla se correlaciona negativamente con la humedad relativa y positivamente con el cacao.

La presencia de los frutales nativos en el mercado está relacionada con el clima, es así que
Lao (2018), refiere que el rendimiento de las plantaciones está relacionado con las variaciones climáticas y los eventos climáticos, es así que un incremento en la temperatura el suelo pierde humedad y el rendimiento baja, además se incrementa la presencia de plagas y enfermedades en las plantaciones. Por su lado, Madrid y Dulanto (2018), observaron que los factores climáticos incidieron positivamente en el rendimiento de frutos en Passiflora.

\section{CONCLUSIONES}

- La mayor abundancia de frutales nativos en el Mercado de Productores 23 de noviembre ocurrió en la época climática seca, presentándose durante todo el periodo de evaluación en cantidades variables siendo las más importantes Myrciaria dubia (Kunth) McVaugh, Solanum sessiliflorum Dunal y Mauritia flexuosa L. f. 
- Los mayores volúmenes de los frutales nativos destacan de manera descendente Solanum sessiliflorum Dunal, Mauritia flexuosa L. f., Myrciaria dubia (Kunth) McVaugh e Inga edulis Mart.

- Los lugares de colecta de los frutales nativos en mayor frecuencia corresponden a la Región Ucayali, Provincia de Coronel Portillo, Distritos de Calleria, Campo Verde y Yarinacocha, caseríos específicos Yerbas buenas, San Juan de Yarinacocha, San José de Yarinacocha, Padre Bernardo y Porvenir.

- Las condiciones climáticas: temperatura $\left({ }^{\circ} \mathrm{C}\right)$ máxima, mínima y media: precipitación (mm) y humedad relativa (\%) se correlacionaron de manera significativa positiva y negativa con los frutales nativos de mayor comercialización y uso como Solanum sessiliflorum Dunal, Mauritia flexuosa L. f., y Oenocarpus bataua Mart..

- Existe un flujo continuo y variable de comercialización de los diferentes frutales nativos que están en función del clima.

\section{RECOMENDACIONES}

- Interés en investigar sobre el manejo agronómico de Mauritia flexuosa "aguaje" y Oenocarpus bataua Mart "ungurahui" debido a que se recoge del medio natural causando presión sobre estas especies.

- Desarrollo de planes de fertilización y manejo integrado de plagas para Solanum sessiliflorum "cocona" y Myrciaria dubia (Kunth) McVaugh "camu camu".

- Propuesta de valor agregado de los frutales nativos en estudio para elevar la rentabilidad de las mismas.

\section{AGRADECIMIENTO}

- A la Agencia Española de Cooperación Internacional para el Desarrollo (AECID) por su contribución en la ejecución de las investigaciones realizadas en las regiones de Apurímac y Ucayali.

- Al Instituto de la Pequeña Producción Sustentable (IPPS) por su financiamiento, asesoramiento, apoyo constante en el desarrollo de la investigación.

\section{REFERENCIAS BIBLIOGRÁFICAS}

Arias Gutiérrez Ruth Irene, Pérez Quintana Manuel Lázaro, Tapia Arias Andrés y Lozano Carpio Pablo. 2018. Cultura alimentaria en comunidades amazónicas. Amazonía Exótica Natural, antrópica, turística. Ecuador. Página 105-124. (On line: https://www.researchgate.net/profile/ Theofilos_Toulkeridis/publication/336702773_ AMAZONIA_EXOTICA_-_Natural_Antropica_ Turistica/links/5dae1e82a6fdccc99d929119/ AMAZONIA-EXOTICA-Natural-Antropica-Turistica.pdf\#page=117 Acceso: 17.10.2020)

Baltazar, O. 2011. Estudio Etnobotánico y de mercado de productos forestales no maderables extraídos del bosque y áreas afines en la ciudad de Pucallpa-Perú.85p.

Brack, A. 2000. Biodiversidad y biocomercio en el Perú.

Brack, A. 2001. La gente y la biodiversidad en la Amazonía peruana. En: Amazonía: orientaciones para el desarrollo sostenible. Lima.

Castillo Torres Myriam Stephanie y Quija Quija Maritza Elizabeth. 2019. Fenología floral de la guaba (Inga edulis) y naranjilla (Solanum quitoense) de acuerdo a la escala $\mathrm{BBCH}$ para uso agroindustrial. Universidad de las Américas, Trabajo de titulación Facultad de Ingeniería y Ciencias Aplicadas: Ingeniería Agroindustrial y de Alimentos. Quito. Ecuador. (On line: http://dspace.udla. edu.ec/bitstream/33000/10715/1/UDLA-ECTIAG-2019-05.pdf Acceso: 17.10.2020)

Dirección Regional Agraria de Ucayali - DRAU. 2012. Plan Estratégico Regional del Sector Agrario de Ucayali 2008 - 2012. Pucallpa. Perú.

FAO. 2008. La Seguridad Alimentaria Mundial: los Desafíos del Cambio Climático y la Bioenergía. Nota informativa. Día Mundial de la Alimentación/TeleFood 2008. (On line: http:// www.fao.org/fileadmin/user_upload/Get_Involved/08WFDINFONOTE_ES.pdf Acceso: 15.10.2020). 
Flores, P.S. 1996. Cultivo de frutales: Manual para el extensionista. Tratado de Cooperación Amazónica-TCA. Secretaría Pro Tempore. Lima. Perú. (On line: http://www.otca-oficial. info/assets/documents/20160704/a57c63090ba9982586b566a2c517ef0a.pdf Acceso: 16.10 .2020$)$.

Flores Flores, J. Miranda Ruíz. E. 2017. Factores que influyen en la rentabilidad económica de la producción del cultivo de camu camu en la Selva Peruana. Revista TZHOECOEN, Vol. 9 Núm. 1 (2017): Tzhoecoen Vol 9 núm 1. (On line: http://revistas.uss.edu.pe/index.php/ tzh/article/view/464 Acceso: 16.10.2020).

Gamboa Auqui, Rafael José. 2019. Caracterización socioeconómica del productor de aguaje (Mauritia Flexuosa I. F.) en la provincia de Leoncio Prado. Universidad Nacional Agraria de la Selva - UNAS. Tesis de posgrado: Maestría en ciencias Agrícolas: mención cultivos tropicales. Tingo María Perú. (On line: http://repositorio.unas.edu.pe/bitstream/handle/UNAS/1666/TS_RJGA_2019. pdf? sequence=1\&isAllowed $=y \quad$ Acceso: 16.10.2020).

Gonzales Coral Agustín. 2007. Frutales nativos amazónicos. Patrimonio alimenticio de la humanidad. IIAP. Iquitos-Perú. 39pp.

Guillén H. W. 2010. Agrobiodiversidad en la Amazonía - conservación. Estación Experimental Agraria Pucallpa. Sub Dirección de Recursos Genéticos y Biotecnología. Pucallpa. Perú.

Huamantupa I. 2008. Frutales nativos silvestres consumidos en los mercados locales y zonas rurales de la Amazonía Peruana (departamentos de Cusco, Loreto y Madre de Dios). Revista Q'EUÑA. 1(2): 026 - 031. Sociedad Botánica del Cusco. Cusco. Perú.

Instituto de Investigación de la Amazonía Peruana (IIAP) 2007. Frutales amazónicos patrimonio de la humanidad. 77p
Lao Zumba Klinton. 2018. Impacto de la variabilidad climática en la economía de los productores de plátano en el distrito de Masisea, provincia de Coronel Portillo, región Ucayali 2017. Universidad Nacional de Ucayali. Tesis para optar el título profesional de: Ingeniero ambiental. Pucallpa. Perú. (On line: http:// repositorio.unu.edu.pe/bitstream/handle/ UNU/3572/000003140T.pdf? sequence $=3 \&$ isAllowed=y Acceso: 17.10.2020).

Madrid Ibarra Flor de María y Dulanto Bejarano Paola Angella. 2018. Impacto de la variabilidad climática y de los sistemas agrarios en el cultivo de granadilla (Passiflora ligularis Juss) de Oxapampa, Pasco, Perú. Biotempo, 2018, 15(1), ene-jun.: 41-48. (On line: http://revistas.urp.edu.pe/index.php/Biotempo/article/ view/1694 Acceso: 17.10.2020).

Mostacero Leon, José; Mejia Coico, Freddy; Gastanadui Rosas, Danilo y De La Cruz Castillo, Jordan. 2017. Inventario taxonómico, fitogreográfico y etnobotánico de frutales nativos del norte del Perú. Scientia Agropecuaria [online]., vol.8, n.3, pp.215-224. ISSN 2077-9917. http://dx.doi.org/10.17268/sci. agropecu.2017.03.04. (On line: http://www. scielo.org.pe/scielo.php?script=sci_arttext\&pid=S2077-99172017000300004 Acceso: 15.10.2020).

Mitjans Moreno Barbarita, Pacheco Escobar Joel y Musaico Lopes Gervásia. 2019. Autogestión comunitaria integral y sostenible de los frutales nativos de Munhiba, Mozambique. Revista Facultad Nacional de Agronomía Medellín; Bogota Tomo 72, N.o 2, (2019): 8829-8840. DOl:10.15446/rfnam. v72n2.78980. (On line: https://search.proquest.com/openview/3ee0464d2b1f30e6f3028ac525b78e66/1?pq-origsite=gscho$|a r \& c b|=2035754$ Acceso: 15.10 .2020 )

Osores Arrascue Hulda Ruth. 2018. Valor económico y cultural de especies de la flora utilizadas en la comunidad aguaruna de Yamayakat, Bagua, Amazonas, Perú. Universidad 
Nacional Mayor de San Marcos. Tesis para optar el Grado Académico de Magíster en Botánica Tropical con Mención en Botánica Económica. (On line: https://cybertesis.unmsm.edu. pe/bitstream/handle/20.500.12672/10307/ Osores_ah.pdf?sequence $=1$ \&isAllowed $=y$ Acceso: 17.10.2020)

Villachica, H. 1996. Frutales y hortalizas promisorias de la Amazonia. SPTTCA № 44. Lima, Perú. p. 243-246.

\section{CORRESPONDENCIA:}

Ing. M Sc. Ena Vilma Velazco Castro

evelazcoc@unia.edu.pe 